\title{
Accelerating Life Test Scheme and Modeling Method for Machinegun
}

\author{
Yong-Hai SHAN, Xian-Ming GAO, Shi-Qi SHAN, Zheng-Gang NIE \\ Baicheng Ordnance Test Center, Baicheng 137001, Jilin, China
}

Received: May 06, 2014 / Accepted: April 13, 2015 / Published: June 25, 2015.

\begin{abstract}
There is a pressing need for improving the current whole life shooting method in order to reduce ammunition consumption in the firearms lifetime test. The influencing factors, the characterization parameters and the correlation analysis result of lifetime were considered. Accelerating life test and measuring scheme for machinegun were designed systematically by applying such theoretical methods as accelerating test, uniform design and lifetime model. As result, it provides a more scientific and reasonable accelerating life test scheme and proposes methods on modeling lifetime, optimizing model and characterizing lifetime. The conclusion can be used in preparing accelerating life test scheme and lifetime model for machinegun.
\end{abstract}

Keywords: Machinegun, barrel, lifetime, design test, model

\section{Introduction}

It is necessary to improve the current whole life shooting method in order to reduce ammunition consumption in the firearms lifetime test ${ }^{[1]}$. In practice, accelerating life method is effective in considerably reducing test consumption. Because barrel is subjected to ablation, corrosion, erosion of high temperature and high pressure propellant gas from the chamber and to bullet abrasion, its environment is harsh. As the main part of machinegun, barrel to some extent represents machinegun in lifetime. Therefore, this paper applies accelerating test, uniform design and statistical analysis to machinegun barrel, proposes accelerating life test scheme and modeling method for machinegun barrel on the basis of massive experimental researches.

\section{Accelerating Life Test Scheme}

Scientific and reasonable accelerating life test scheme is based on correlation theories, lots of tests and experimental verification.

By massive theoretical researches and tests, we find

Corresponding author: Yong-Hai SHAN, Baicheng Ordnance Test Center, Baicheng 137001, Jilin, China. E-mail: yhsh68@163.com. that the main controllable factors inflecting the machinegun barrel lifetime are test temperature, bullet consumption within a cooling cycle (BCCC) and interval time between changing ammunition boxes (ITCAB), and confirm that the characterization parameters of machinegun barrel lifetime are abrasion in chamber, initial velocity of bullet, pressure in chamber, firing dispersion, and so ${ }^{[3]}{ }^{[4]}$. Under given shooting specifications, lifetime index and correlation ballistic parameters, it is easy to calculating the consumption bullets of machinegun lifetime. However, the more important is deciding the relationship between main controllable factors and machinegun lifetime (see Fig.1). If we find the independent and mutual action rules of the main factors (test temperature, BCCC and ITCAB) on lifetime, and establish corresponding mathematical model, then it is not difficult to predict the machinegun lifetime under any combination of shooting specifications. Therefore, four kinds of test schemes are proposed as follows (see Tab.1 and Fig.2):

Scheme one: Studying on the rule of ITCAB on lifetime with test temperature and $\mathrm{BCCC}$ being constants. 
Scheme two: Studying on the rule of test temperature on lifetime with ITCAB and BCCC being constants.

Scheme three: Studying on the rule of BCCC on lifetime with test temperature and ITCAB being constants.

Scheme four: Improving and applying Latin square uniform design ${ }^{[5]}[6]$, taking small samples and design uniformity into account, optimizing lifetime study scheme, and studying mutual action rule of test temperature, BCCC and ITCAB on lifetime.

\section{Modeling Method}

Test according to the four schemes in the Tab.1 and measure or calculate interior and exterior ballistic parameters as temperature of the outer wall of barrel, erosion in chamber, and so on when barrel has shot a certain amount of bullet. See Tab.2 (it is for test scheme one, other parameters measurement schemes are similar to this one.)for the parameters measurement scheme. These parameters are the base of analyzing lifetime function and setting up lifetime model.

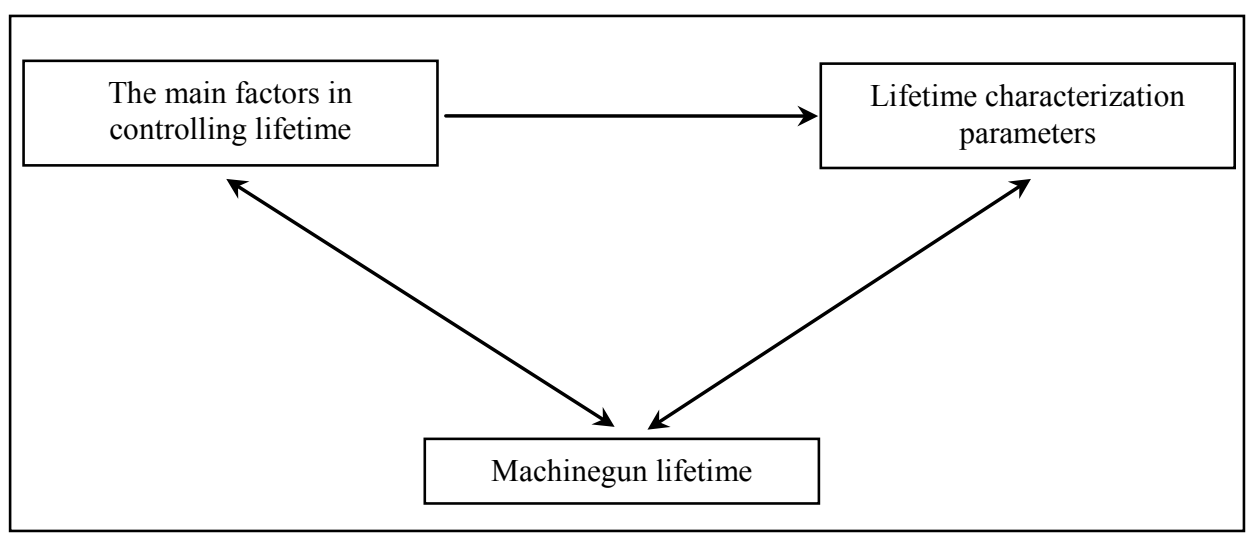

Fig.1 process of getting machinegun lifetime.

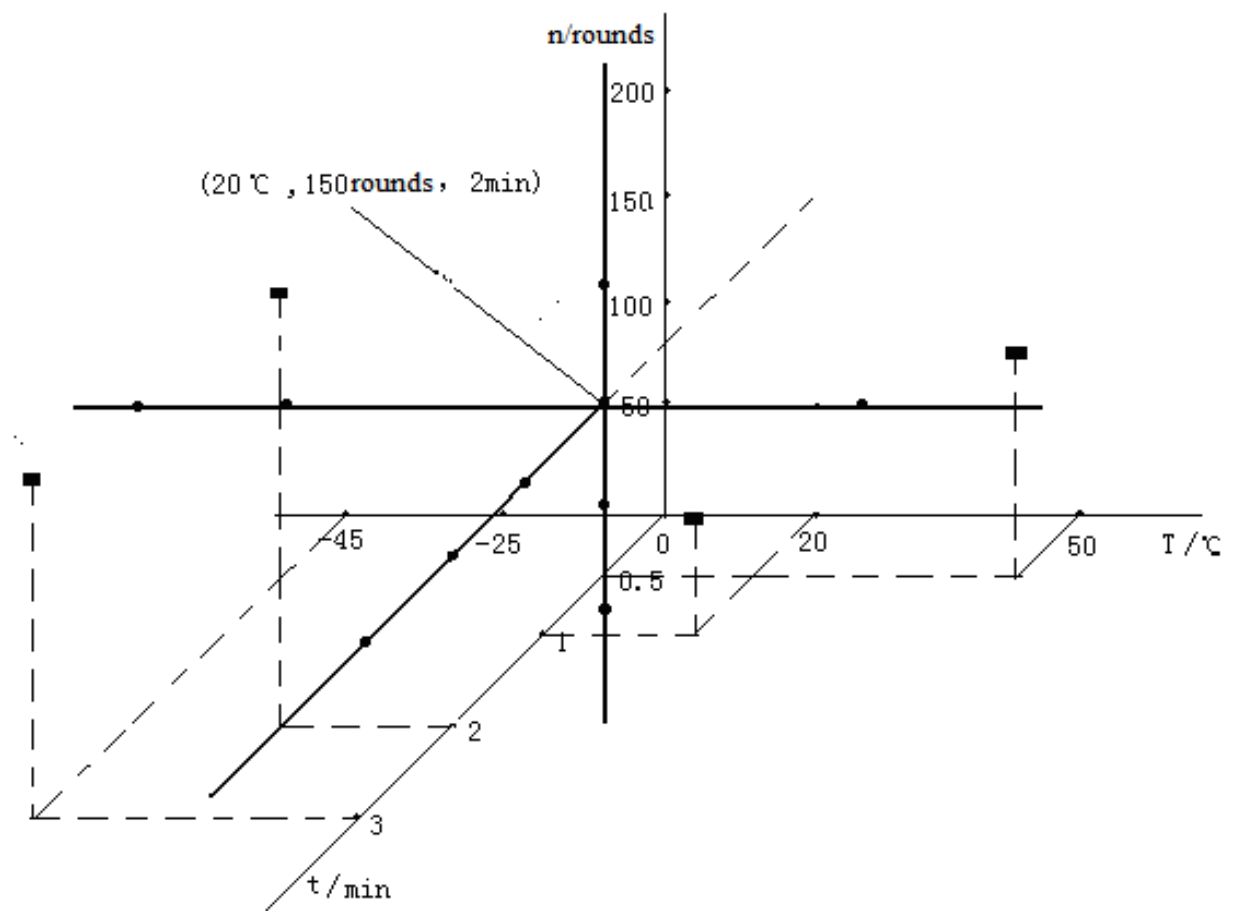

Fig. 2 distribution of three-factor independent action points (dots on the three axes) and mutual action points (black boxes). 
Table 1 Test Scheme.

\begin{tabular}{|c|c|c|c|c|}
\hline $\begin{array}{l}\text { scheme } \\
\text { number }\end{array}$ & barrel number & $\begin{array}{l}\text { environment } \\
\text { temperature }\left({ }^{\circ} \mathrm{C}\right)\end{array}$ & BCCC (round) & ITCAB (min) \\
\hline \multirow{4}{*}{ one } & 1 & \multirow{4}{*}{20} & $25 \times 2 \times 3$ & 0.5 \\
\hline & 2 & & $25 \times 2 \times 3$ & 1 \\
\hline & 3 & & $25 \times 2 \times 3$ & 2 \\
\hline & 4 & & $25 \times 2 \times 3$ & 3 \\
\hline \multirow{4}{*}{ two } & 5 & 50 & $25 \times 2 \times 3$ & 2 \\
\hline & $6(3)$ & 22 & $25 \times 2 \times 3$ & 2 \\
\hline & 7 & -25 & $25 \times 2 \times 3$ & 2 \\
\hline & 8 & -45 & $25 \times 2 \times 3$ & 2 \\
\hline \multirow{4}{*}{ three } & 9 & \multirow{4}{*}{20} & $25 \times 2 \times 1$ & 2 \\
\hline & 10 & & $25 \times 2 \times 2$ & 2 \\
\hline & $11(3)$ & & $25 \times 2 \times 3$ & 2 \\
\hline & 12 & & $25 \times 2 \times 4$ & 2 \\
\hline \multirow{4}{*}{ four } & 13 & -45 & $25 \times 2 \times 3$ & 3 \\
\hline & 14 & -25 & $25 \times 2 \times 4$ & 2 \\
\hline & 15 & 20 & $25 \times 2 \times 1$ & 1 \\
\hline & 16 & 50 & $25 \times 2 \times 2$ & 0.5 \\
\hline
\end{tabular}

$\mathrm{BCCC}=$ burst length $\times$ burst firing times $\times$ amount of ammunition boxes

Table 2 comprehensive parameters test scheme for scheme one.

\begin{tabular}{|c|c|c|c|c|c|c|c|c|c|c|}
\hline $\begin{array}{l}\text { Barrel } \\
\text { No. }\end{array}$ & $\begin{array}{l}\text { environment } \\
\text { temperature } \\
\left({ }^{\circ} \mathrm{C}\right)\end{array}$ & $\begin{array}{l}\text { BCCC } \\
\text { (round) }\end{array}$ & $\begin{array}{l}\text { ITCAB } \\
\text { (min) }\end{array}$ & $\begin{array}{l}\text { cumulative } \\
\text { amount of } \\
\text { bullet (rounds) }\end{array}$ & $\begin{array}{l}\text { max barrel } \\
\text { temperature } \\
\text { after shooting } \\
\left({ }^{\circ} \mathrm{C}\right)\end{array}$ & $\begin{array}{l}\text { initial } \\
\text { velocity } \\
(\mathrm{m} / \mathrm{s})\end{array}$ & $\begin{array}{l}\text { firing } \\
\text { dispersion } \\
(\mathrm{cm})\end{array}$ & $\begin{array}{l}\max \\
\text { chamber } \\
\text { pressure } \\
(\mathrm{MPa})\end{array}$ & $\begin{array}{l}\text { erosion in } \\
\text { chamber } \\
(\mathrm{mm})\end{array}$ & $\begin{array}{l}\text { lifetime } \\
\text { (round) }\end{array}$ \\
\hline \multirow{4}{*}{1} & \multirow{4}{*}{20} & \multirow{4}{*}{$25 \times 2 \times 3$} & \multirow{4}{*}{0.5} & 600 & - & - & - & - & - & \multirow{4}{*}{-} \\
\hline & & & & 1200 & - & - & - & - & - & \\
\hline & & & & 1800 & - & - & - & - & - & \\
\hline & & & & 2400 & - & - & - & - & - & \\
\hline \multirow{4}{*}{2} & \multirow{4}{*}{20} & \multirow{4}{*}{$25 \times 2 \times 3$} & \multirow{4}{*}{1} & 600 & - & - & - & - & - & \multirow{4}{*}{-} \\
\hline & & & & 1200 & - & - & - & - & - & \\
\hline & & & & 1800 & - & - & - & - & - & \\
\hline & & & & 2400 & - & - & - & - & - & \\
\hline \multirow{4}{*}{3} & \multirow{4}{*}{20} & \multirow{4}{*}{$25 \times 2 \times 3$} & \multirow{4}{*}{2} & 600 & - & - & - & - & - & \multirow{4}{*}{-} \\
\hline & & & & 1200 & - & - & - & - & - & \\
\hline & & & & 1800 & - & - & - & - & - & \\
\hline & & & & 2400 & - & - & - & - & - & \\
\hline \multirow{4}{*}{4} & \multirow{4}{*}{20} & \multirow{4}{*}{$25 \times 2 \times 3$} & \multirow{4}{*}{3} & 600 & - & - & - & - & - & \multirow{4}{*}{-} \\
\hline & & & & 1200 & - & - & - & - & - & \\
\hline & & & & 1800 & - & - & - & - & - & \\
\hline & & & & 2400 & - & - & - & - & - & \\
\hline
\end{tabular}

According to the index and the change trend of each barrel's initial velocity $\mathrm{v}$, dispersion $\mathrm{R}_{50}$, chamber pressure $\mathrm{P}$ and erosion in chamber $\mathrm{d}$ with cumulative amount of shooting bullet, we can work out the lifetime $\mathrm{n}$ of each barrel in different combinations of test temperature, $\mathrm{BCCC}$ and ITCAB. 


$$
\begin{gathered}
n=\min \left(n_{1}, n_{2}, n_{3}, n_{4}\right) \\
n_{1}=f(v), n_{2}=\varphi\left(R_{50}\right), n_{3}=\phi(P), n_{4}=\vartheta(d)
\end{gathered}
$$

Among them, $\mathrm{n}$ is lifetime. $\mathrm{n} 1, \mathrm{n} 2, \mathrm{n} 3$ and $\mathrm{n} 4$ are lifetimes determined by initial velocity $\mathrm{v}$, dispersion $\mathrm{R}_{50}$, chamber pressure $\mathrm{P}$ and erosion in chamber $\mathrm{d}$ respectively.

Therefore, every barrel lifetime in different schemes can be acquired. Then, the one-factor, two-factor and three-factor models in which barrel lifetime changes with test temperature, $\mathrm{BCCC}$ and ITCAB are as follows:

Scheme one: $n=n(x)$; Scheme two: $n=n(y)$; Scheme three: $n=n(z)$; Scheme four: $n=n(x, y, z)$

Among them, $\mathrm{x}$ is test environment temperature, $\mathrm{y}$ is $\mathrm{BCCC}$ and $\mathrm{z}$ is ITCAB.

Thus, preliminarily establish three types of models.

\subsection{Polynomial Regression Model}

Three types of polynomial regression models are chief considerations as the three influential factors and their individual or mutual influence to the lifetime have been analyzed and determined.

(1) Single Stress Model

Its main concern is individual influence from test temperature, BCCC and ITCAB on lifetime.

$$
n=a x^{2}+b x+c^{[7]}
$$

(2) Double Stress Model

Its main concern is mutual influence of two factors in test temperature, BCCC and ITCAB on lifetime.

$$
\begin{aligned}
& n=a x^{2}+b x y+c y^{2}+d x z+e y z \\
& +f z^{2}+g x+h y+i z+j
\end{aligned}
$$

(3) Three Stress Model

Its main concern is mutual influence among test temperature, $\mathrm{BCCC}$ and ITCAB on lifetime.

$$
\begin{aligned}
& n=a x^{3}+b y^{3}+c z^{3}+d x^{2} y+e x y^{2} \\
& +f x^{2} z+g z^{2}+h y^{2} z+i y z^{2}+j x y z \\
& +k x y+l x z+m y z+n x+o y+p z+q
\end{aligned}
$$

Among them, $\mathrm{n}$ represents lifetime; $\mathrm{x}, \mathrm{y}$ and $\mathrm{z}$ represent various stresses; a, b, c... q represent stress coefficient.

The model can be simplified according to Forward Regression, Backward Regression, optional variables and experience.

\subsection{Accelerating Test Model}

\section{(1) Single Stress Model}

The expression methods are more sophisticated both in Arrhenius model $n=A e^{E / K T}$ whose accelerated variable is temperature, and in Inverse Power Law model $n=1 /\left(\mathrm{KV}^{\mathrm{C}}\right)$ whose accelerated variable is pressure $^{[8]-[12]}$. In order to facilitate the identification and acquisition parameters, these methods can be simplified as $I n n=a+b / X$ or $\operatorname{In} n=a+b \times \varphi(x)$ through proper transformation.

According to the principle of Least Squares, if:

$$
\begin{gathered}
G=\sum_{i=1}^{k}\left(\ln n_{i}-a-b / X_{i}\right)^{2}, \\
\left\{\begin{array}{l}
\frac{\partial G}{\partial a}=-2 \sum_{i=1}^{k}\left(\ln n_{i}-a-b / X_{i}\right)=0 \\
\frac{\partial G}{\partial b}=-\sum_{i=1}^{k} \frac{2}{X_{i}}\left(\ln n_{i}-a-b / X_{i}\right)=0
\end{array}\right.
\end{gathered}
$$

then, we can get the formula for identifying parameter in single stress model as follow:

$$
\left\{\begin{array}{c}
\hat{a}=\frac{\sum_{i=1}^{k} 1 / T_{i} \sum_{i=1}^{k} \ln n_{i} / X_{i}-\sum_{i=1}^{k} 1 / X_{i}^{2} \sum_{i=1}^{k} \ln n_{i}}{\left(\sum_{i=1}^{k} 1 / X_{i}\right)^{2}-k \sum_{i=1}^{k} 1 / X_{i}^{2}} \\
\hat{b}=\frac{\sum_{i=1}^{k} 1 / X_{i} \sum_{i=1}^{k} \ln n_{i}-k \sum_{i=1}^{k} \ln n_{i} / X_{i}}{\left(\sum_{i=1}^{k} 1 / X_{i}\right)^{2}-k \sum_{i=1}^{k} 1 / X_{i}^{2}}
\end{array}\right.
$$

(2) Double Stress Model

$$
n=\frac{A}{V^{C}} \cdot e^{\frac{B}{T}} \text { and } n=\eta_{0}\left(\frac{v_{0}}{v}\right)^{\alpha}\left(\frac{p_{0}}{p}\right)^{\beta} \text { are common }
$$

double stresses accelerating life models ${ }^{[8]-[12]}$. Calculating parameters in these expression methods is more complex. For the same reason, these methods can be simplified as $\ln n=a+b / X_{1}+c / X_{2}$ or $\ln n=a+b \phi\left(x_{1}\right)+c \vartheta\left(x_{2}\right)$. 
According to the principle of Least Squares, if:

$$
\begin{aligned}
& G=\sum_{i=1}^{k}\left(\ln n_{i}-a-b / X_{1 i}-c / X_{2 i}\right)^{2}, \\
& \left\{\begin{array}{l}
\frac{\partial G}{\partial a}=-2 \sum_{i=1}^{k}\left(\ln n_{i}-a-b / X_{1 i}-c / X_{2 i}\right)=0 \\
\frac{\partial G}{\partial b}=-\sum_{i=1}^{k} \frac{2}{X_{1 i}}\left(\ln n_{i}-a-b / X_{1 i}-c / X_{2 i}\right)=0 \\
\frac{\partial G}{\partial c}=-\sum_{i=1}^{k} \frac{2}{X_{2 i}}\left(\ln n_{i}-a-b / X_{1 i}-c / X_{2 i}\right)=0
\end{array}\right. \\
& A=\sum_{i=1}^{k} \ln n_{i} \cdot \sum_{i=1}^{k} \frac{1}{X_{1 i}^{2}} \cdot \sum_{i=1}^{k} \frac{1}{X_{2 i}^{2}}+\sum_{i=1}^{k} \frac{1}{X_{1 i}} \cdot \sum_{i=1}^{k} \frac{1}{X_{2 i}} \ln n_{i} \cdot \sum_{i=1}^{k} \frac{1}{X_{1 i} X_{2 i}}+\sum_{i=1}^{k} \frac{1}{X_{2 i}} \cdot \sum_{i=1}^{k} \frac{1}{X_{1 i}} \ln n_{i} \cdot \sum_{i=1}^{k} \frac{1}{X_{1 i} X_{2 i}} \\
& -\sum_{i=1}^{k} \frac{1}{X_{2 i}} \cdot\left(\sum_{i=1}^{k} \frac{1}{X_{1 i}}\right)^{2} \cdot \sum_{i=1}^{k} \frac{1}{X_{2 i}} \ln n_{i}-\sum_{i=1}^{k} \frac{1}{X_{1 i}} \cdot \sum_{i=1}^{k} \frac{1}{X_{1 i}} \ln n_{i} \cdot \sum_{i=1}^{k} \frac{1}{X_{2 i}^{2}}-\sum_{i=1}^{k} \ln n_{i} \cdot\left(\sum_{i=1}^{k} \frac{1}{X_{1 i} X_{2 i}}\right)^{2} \\
& B=k \sum_{i=1}^{k} \frac{1}{X_{2 i}^{2}} \cdot \sum_{i=1}^{k} \frac{1}{X_{1 i}} \ln n_{i}+\sum_{i=1}^{k} \frac{1}{X_{2 i}} \cdot \sum_{i=1}^{k} \frac{1}{X_{1 i} X_{2 i}} \cdot \sum_{i=1}^{k} \ln n_{i}+\sum_{i=1}^{k} \frac{1}{X_{1 i}} \cdot \sum_{i=1}^{k} \frac{1}{X_{2 i}} \cdot \sum_{i=1}^{k} \frac{1}{X_{2 i}} \ln n_{i} \\
& -\left(\sum_{i=1}^{k} \frac{1}{X_{2 i}}\right)^{2} \cdot \sum_{i=1}^{k} \frac{1}{X_{1 i}} \ln n_{i}-\sum_{i=1}^{k} \frac{1}{X_{1 i}} \cdot \sum_{i=1}^{k} \frac{1}{X_{2 i}^{2}} \cdot \sum_{i=1}^{k} \ln n_{i}-k \sum_{i=1}^{k} \frac{1}{X_{1 i} X_{2 i}} \cdot \sum_{i=1}^{k} \frac{1}{X_{2 i}} \ln n_{i} \\
& C=k \sum_{i=1}^{k} \frac{1}{X_{1 i}^{2}} \cdot \sum_{i=1}^{k} \frac{1}{X_{2 i}} \ln n_{i}+\sum_{i=1}^{k} \frac{1}{X_{1 i}} \cdot \sum_{i=1}^{k} \frac{1}{X_{2 i}} \cdot \sum_{i=1}^{k} \frac{1}{X_{1 i}} \ln n_{i}+\sum_{i=1}^{k} \frac{1}{X_{1 i}} \cdot \sum_{i=1}^{k} \frac{1}{X_{1 i} X_{2 i}} \cdot \sum_{i=1}^{k} \ln n_{i} \\
& -\sum_{i=1}^{k} \frac{1}{X_{1 i}^{2}} \cdot \sum_{i=1}^{k} \frac{1}{X_{2 i}} \cdot \sum_{i=1}^{k} \ln n_{i}-\left(\sum_{i=1}^{k} \frac{1}{X_{1 i}}\right)^{2} \cdot \sum_{i=1}^{k} \frac{1}{X_{2 i}} \ln n_{i}-k \sum_{i=1}^{k} \frac{1}{X_{1 i}} \cdot \sum_{i=1}^{k} \frac{1}{X_{1 i} X_{2 i}} \\
& D=k \sum_{i=1}^{k} \frac{1}{X_{1 i}^{2}} \cdot \sum_{i=1}^{k} \frac{1}{X_{1 i} X_{2 i}}+2 \sum_{i=1}^{k} \frac{1}{X_{1 i}} \cdot \sum_{i=1}^{k} \frac{1}{X_{2 i}} \cdot \sum_{i=1}^{k} \frac{1}{X_{1 i} X_{2 i}}-\left(\sum_{i=1}^{k} \frac{1}{X_{2 i}}\right)^{2} \cdot \sum_{i=1}^{k} \frac{1}{X_{1 i}^{2}}- \\
& k\left(\sum_{i=1}^{k} \frac{1}{X_{1 i} X_{2 i}}\right)^{2}-\left(\sum_{i=1}^{k} \frac{1}{X_{1 i}}\right)^{2} \cdot \sum_{i=1}^{k} \frac{1}{X_{2 i}^{2}}
\end{aligned}
$$

\subsection{Temperature Field Model}

(1) Predicting barrel lifetime model on the basis of fatigue lifetime between barrel chromium plating layer and the base

Barrel fatigue can be considered as low period fatigue by heat and chamber pressure. Barrel lifetime can be calculated through Manson-Coffin fatigue formula $^{[13][14]}$.

$$
\frac{\Delta \varepsilon_{p}}{2}=\varepsilon_{f}^{\prime} \cdot(n)^{c}
$$

In this formula: $\frac{\Delta \varepsilon_{p}}{2}$ is plastic strain; $\varepsilon_{f}^{\prime}$ is material fatigue ductility coefficient; $\mathrm{n}$ is load cycles, i.e. barrel lifetime; $c$ is material fatigue ductility exponent, its range is $-0.8 \sim-0.3$.

(2) Predicting barrel lifetime model on the basis of thermal effects

The erosion in chamber gradually increases in certain rule with barrel failure [15-18] (see Fig.3).

Based on test data, empirical formula for calculating one bullet ablation in chamber is:

$$
W=A e^{b T_{w}} \times 10^{-4}
$$

In the formula: $\mathrm{w}$ is ablation in chamber $(\mathrm{mm})$; A and $b$ are empirical constants; $T_{W}$ is chamber wall temperature.

Chamber wall temperature after shooting one bullet is :

$$
T_{w}=1.096\left(T_{f}-T_{c}-600\right) \cdot \sqrt{\omega \cdot p_{m}} / d
$$




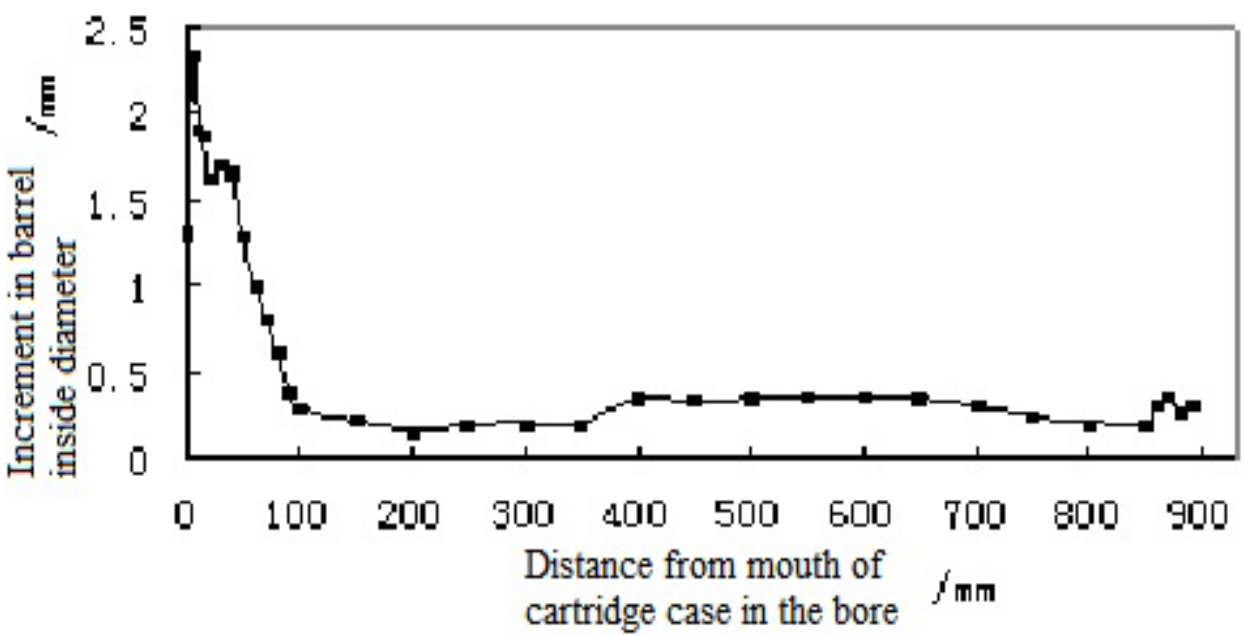

Fig. 3 Increment in barrel inside diameter in a machinegun.

Chamber wall temperature after continuous shooting is :

$$
T_{t}=0.4632\left(T_{f}-T_{c}-600\right) \omega^{0.75} \sqrt{(N-1) R / d}
$$

In the formula: $\mathrm{T}_{\mathrm{f}}$ is propellant explosion temperature $(\mathrm{K}) ; \mathrm{T}_{\mathrm{c}}$ is correlated temperature when additives are used $(\mathrm{K})$; $\mathrm{d}$ is caliber $(\mathrm{mm}) ; \omega$ is charge amount $(\mathrm{kg}) ; \mathrm{p}_{\mathrm{m}}$ is the maximum pressure in chamber (MPa); $\mathrm{N}$ is firing bullet amount; $\mathrm{R}$ is fire rate.

If the maximum erosion in chamber reaches $5 \%$ of the land diameter, the barrel life is end. Calculating cumulative erosion in chamber according to the above-mentioned formulae, then the barrel lifetime can be predicted.

\subsection{Optimum Model}

The better model whose error is small, stability is good and which is convenient to model can be selected out by experimental verification, error analysis and model stability analysis.

\section{Conclusions}

Through comprehensive analysis and research, this paper gets results as follows:

(1) Optimized the machinegun accelerating life test scheme by applying accelerating test and uniform design method; proposed four accelerating life test schemes and measuring parameters scheme which considered the influence of single factor and multi factors;

(2) Based on experimental research, proposed three types of modeling lifetime methods, simplifying model methods, identifying parameters methods and model expressions according to the relationship among the main influence factors on machinegun barrel lifetime, lifetime characterization parameters and lifetime.

The research result is a guideline on theory and method in preparing machinegun accelerating life test scheme, modeling lifetime, predicting lifetime and applying in test.

\section{References}

[1] MA Li, et al. GJB3484-98 Test Method for Firearms Characterization [s]

[2] SHEN Jin-xing. Firearms Lifetime [M]. Taiyuan: Shanxi United University Press, 1994

[3] SHAN Yong-hai, et al. Study on Lifetime Test for Machinegun Barrel in Normal Temperature[J]. Beijing: ACTA ARMAMENTARII. 2013, 34(1):1-3

[4] SHAN Yong-hai, et al. Technical Analysis of Relativity of Shooting Criterions and Firearms Lifetime[J]. Nanjing: Journal of Nanjing University of Science and Technology,2005, 29(4):417-424

[5] FANG Kai-tai. Uniform Design and Uniform Design Table [M]. Beijing: Science Press,1994

[6] FANG Kai-tai, MA Chang-xing. Orthogonal and 
Uniform Design[M]. Hong Kong: Hong Kong Baptist University, 2000

[7] YUAN Zhi-fa, ZHOU Jing-yu. Design and Analysis of Experiments[M]. Beijing: Higher Education Press, 2000

[8] ZHANG Chun-hua, WEN Xi-sen, CHEN Xun. Summarization of accelerating life test[J]. Beijing: ACTA ARMAMENTARII[J]. 2004, 25(4):485-488

[9] MAO Shi-song. From lifetime test to accelerating life test[J]. Quality and reliability.2003,(1): 8-12

[10] LIU Lu-sheng, WAN Min-kan. Research on accelerating life test method[J]. Ordnance Test. 2000,(4):29-34

[11] MAO Shi-song, WANG Ling-ling. Accelerating lifetime test[M]. Beijing: Science Press, 1997

[12] ZHAO Shao-bian. Anti Fatigue Design[M]. Beijing: China Machine Press, 1994

[13] ZHAO Shao-bian, WANG Zhong-bao. Anti Fatigue
Design- methods and data[M]. Beijing: China Machine Press, 1997

[14] WU Yong-Hai, XU Cheng, LI Feng. Thermal Effect of Large-calibre Machine-gun Barrel Subjected to Thermal Shock[J]. Nanjing: Journal of Nanjing University of Science and Technology, 2007(1):1-5

[15] GAO Fu-shen, QIU Wen-jian, LIANG Shi-chao, et at. Study on the Gun Tube Life Prediction. Journal of Nanjing University of Science and Technology, 1997, (03):229-232.

[16] WU Bin, XIA Wei, TANG Yong, et al. Prediction Model on Gun Tube Melting and Ablation. Gun Launch \& Control Journal, 2002(1):5-10

[17] LI Ming-tao, CUI Wan-shan, YAO Zhe, et al. Predication Method of Barrel Life Based on Inner Side Melting Theory. Gun Launch \& Control Journal, 2009(2):5-8 